\title{
NUEVAS CITAS PARA LA FLORA DE LA REGIÓN DE BAHÍA BLANCA (PROVINCIA DE BUENOS AIRES, ARGENTINA)
}

\author{
MARÍA E. GIL ${ }^{1}$, ANA C. ANDRADA ${ }^{1} \&$ CECILIA N. PELLEGRINI ${ }^{1}$
}

\begin{abstract}
Summary: Gil, M. E., A. C. Andrada \& C. N. Pellegrini. 2012. New records for the flora of Bahía Blanca region (Buenos Aires province, Argentina). Bonplandia 21(2): 135-137.

In this paper we provide new data on the distribution of two species of Poaceae in the Buenos Aires province, Argentina.
\end{abstract}

Key words: Erioneuron pilosum var. mendocinum; Nassella sanluisensis; Bahía Blanca.

Resumen: Gil, M. E., A. C. Andrada \& C. N. Pellegrini. 2012. Nuevas citas para la flora de la región de Bahía Blanca (provincia de Buenos Aires, Argentina). Bonplandia 21(2): 135-137.

En el presente trabajo se aportan nuevos datos sobre la distribución de dos especies de Poaceae en la provincia de Buenos Aires, Argentina.

Palabras clave: Erioneuron pilosum var. mendocinum; Nassella sanluisensis; Bahía Blanca.

\section{Introducción}

En el marco de un estudio sobre los atributos florísticos de un área en proceso de ser declarada Reserva Natural Urbana, surgió el hallazgo de dos nuevas referencias de Poaceae para los alrededores de la ciudad de Bahía Blanca, provincia de Buenos Aires. El sitio constituye un verdadero ecotono con representantes vegetales de las provincias fitogeográficas Pampeana, del Espinal, del Monte y Patagónica (Gil et al., 2011). El objetivo de este trabajo es dar a conocer la existencia de Erioneuron pilosum var. mendocinum y Nassella sanluisensis en el partido de Bahía Blanca. La primera constituye el primer registro para la provincia de Buenos Aires, mientras que la segunda, si bien se encuentra extensamente distribuida en
Argentina, no había sido citada previamente para el partido de Bahía Blanca (Zuloaga et al., 2008; Anton et al., 2010).

\section{Materiales y Métodos}

Se realizaron expediciones periódicas durante los años 2009-2011 al sitio conocido como Cueva de los Leones (38 $38^{\circ} \mathrm{S}, 62^{\circ} 17^{\prime} \mathrm{O}$ ), a $11 \mathrm{~km}$ de la ciudad de Bahía Blanca en el SO del distrito Austral de la Provincia Fitogeográfica Pampeana. Consiste en una escarpa erosiva situada en el extremo distal de la llanura Surventánica, que descansa en la llanura marítima subyacente (González Uriarte, 2010). El paisaje exhibe compartimentos ecológicos relacionados y definidos como

\footnotetext{
${ }^{1}$ Laboratorio de Sistemática Vegetal, Departamento de Agronomía, Universidad Nacional del Sur, San Andrés 800,
} 8000 Bahía Blanca, Prov. de Buenos Aires, Argentina. Tel.: 54291 4595102. E-mail: megil@uns.edu.ar 
planicies que limitan con terrazas rocosas, las cuales continúan en barrancas donde se observan cuevas y cañadas. Éstas desembocan en el arroyo Saladillo de García Dulce, distante a ca. $1 \mathrm{~km}$ de la formación. La fisonomía de la vegetación corresponde a una estepa arbustiva con un estrato herbáceo donde predominan los pastos. Los especímenes recolectados están depositados en el Herbario Regional del Departamento de Agronomía de la Universidad Nacional del Sur (BB).

\section{Tratamiento taxonómico}

\section{Erioneuron pilosum (Buckley) Nash var. mendocinum (Parodi) Nicora, Darwiniana 18: 104.1973.}

\section{Sinonimia en Anton et al., 2010}

Se confirma para la provincia de Buenos Aires esta especie (Tribu Eragrostideae) no citada previamente por Cabrera (1970).

Distribución geográfica: Parodi (1937) la halló formando una isleta en la provincia de Mendoza, mientras que Nicora (1978) la citó para la precordillera de dicha provincia. Zuloaga et al. (2008) restringen su presencia a las provincias de La Pampa y Mendoza. Rúgolo de Agrasar et al. (2005) la consideran un endemismo de las provincias de Mendoza y La Pampa, donde vive en Lihuel Calel, en las barrancas del sudeste y sobre basaltos, en el noroeste.

Iconografía: Parodi, Revista Argentina de Agronomía 4(4): 251, Fig. 2 A y B, 1937.

Material examinado: ARGENTINA. Prov. Buenos Aires: Partido Bahía Blanca, Cueva de los Leones, 2-12-2009 Gil et al., 5331 (BB).

Observaciones: En Buenos Aires crece sobre planchas rocosas, formando manchones discretos y aislados.

\section{Nassella sanluisensis (Speg.) Barkworth Taxon 39 (4): 611. 1990.}

Sinonimia en Anton et al., 2010
Se amplía la zona de distribución de esta especie no incluida en la Flora de la Provincia de Buenos Aires (Cabrera, 1970) ni citada para la región de Bahía Blanca por Lamberto et al. (1997).

Distribución geográfica: La primera cita para la provincia de Buenos Aires fue realizada por Torres (1993) con materiales provenientes de los partidos de Villarino, Tornquist y Cnel. Suárez. También existe registro de, al menos, un ejemplar coleccionado en el año 2010 en el partido de Adolfo Alsina (Buenos Aires), depositado en el herbario del Instituto de Botánica Darwinion, (Zuloaga et al. 12684 (SI)). Para su distribución en Argentina Zuloaga et al. (2008) y Anton et al. (2010) incluyen las provincias de Buenos Aires, Catamarca, Córdoba, Jujuy, La Pampa, La Rioja, Mendoza, Neuquén, Río Negro, Santiago del Estero, San Juan y San Luis.

Iconografía: Rúgolo de Agrasar, Steibel \& Troiani, Manual Ilustrado de las Gramíneas de la provincia de La Pampa: 148, 2005.

Material examinado: ARGENTINA. Prov. Buenos Aires: Partido Bahía Blanca, Cueva de los Leones, 2-12-2009 Gil et al., 5332 (BB).

Observaciones: En Buenos Aires crece en sectores de suelo con roca aflorante.

\section{Agradecimientos}

Los autores desean expresar suagradecimiento al Ing. Sergio A. Lamberto por la inestimable colaboración durante la revisión de los materiales coleccionados y la lectura del manuscrito. Asimismo, al Dr. Roberto Tortosa (Herbario BAA), Mus. Marisa Prada (Herbario LP), Dr. Walter Muiño (Herbario SRFA), Ing. Agr. Eduardo Méndez (Herbario MERL), Dra. Mirta Arriaga (Herbario BA) y Lic. Walter Medina (Herbario CTES) por la información brindada.

\section{Bibliografía}

ANTON, A. M., Z. RÚGOLO DE AGRASAR \& F. 
O. ZULOAGA. 2010. Flora Argentina. Tomo 3 Monocotyledoneae, Poaceae. Consultado Agosto 2012. Website: http://www.floraargentina.edu.ar

CABRERA, A. L. 1970. Flora de la Provincia de Buenos Aires. Parte II - Gramíneas. Colección Científica del INTA, Buenos Aires. 624 pp.

GIL, M. E., A. C.ANDRADA \& C. N. PELLEGRINI. 2011. Atributos florísticos de la Cueva de los Leones, Bahía Blanca, Argentina. Resúmenes de las III Jornadas Argentinas de Ecología de Paisajes. Website: http:// www.asadep.org.ar/documentos/IIIJAEP/Resumenes. pdf

GONZÁLEZ URIARTE, M. 2010. Geomorfología aplicada a la gestión ambiental. En: PAOLONI, J. D. Ambientes y recursos naturales del partido de Bahía Blanca. EdiUNS, Bahía Blanca: 89-126.

LAMBERTO, S. A., A. F. VALLE, E. M. ARAMAYO \& A. C. ANDRADA. 1997. Manual Ilustrado de las plantas silvestres de la región de Bahía Blanca. Departamento de Agronomía, Universidad Nacional del Sur Ed. Bahía Blanca, Argentina. 548 pp.
NICORA, E. G. 1978. Gramineae. En: CORREA, M. N. Flora Patagónica. Parte III. Colección Científica INTA. 563 pp.

PARODI, L. R. 1937. Revisión de las gramíneas del género Tridens de la flora argentina. Revista Argentina de Agronomía, 4: 241-257

RÚGOLO DE AGRASAR, Z. E., P. E. STEIBEL \& H. O. TROIANI. 2005. Manual Ilustrado de las Gramíneas de la provincia de La Pampa. $1^{\circ}$ ed. La Pampa, Santa Rosa: Editorial de la Universidad Nacional de La Pampa; Córdoba, Río Cuarto: Editorial de la Universidad Nacional de Río Cuarto. 359 pp.

TORRES, M. A. 1993. Revisión del género Stipa (Poaceae) en la provincia de Buenos Aires. Monografía 12, Comisión de Investigaciones Científicas de la provincia de Buenos Aires. La Plata, Argentina. 62 pp.

ZULOAGA, F. O., O. MORRONE \& M. J. BELGRANO. 2008 en adelante. Catálogo de las Plantas Vasculares del Cono Sur. Instituto de Botánica Darwinion. Versión Junio 2011. Website: http://www2.darwin. edu.ar/Proyectos/FloraArgentina/FA.asp

Original recibido el 29 de junio de 2012; aceptado el 17 de septiembre de 2012. 
